International Mathematical Forum, Vol. 9, 2014, no. 17, 825 - 833

HIKARI Ltd, www.m-hikari.com

http://dx.doi.org/10.12988/imf.2014.4465

\title{
Ricci Generalized Pseudo-Parallel Legendrian Submanifolds with Flat Normal Bundle of Sasakian Space Forms
}

\author{
Xiaomin Chen ${ }^{1}$ \\ College of Sciences \\ China University of Petroleum (Beijing) \\ Beijing, 102249, China
}

Copyright (c) 2014 Xiaomin Chen. This is an open access article distributed under the Creative Commons Attribution License, which permits unrestricted use, distribution, and reproduction in any medium, provided the original work is properly cited.

\begin{abstract}
Let $\widetilde{M}^{2 n+1}(c)$ be a $2 n+1$-dimensional Sasakian space form with constant $\phi$-sectional curvature $c \neq-3$ and $M^{n}$ be a Legendrian submanifold with flat normal bundle of $\widetilde{M}^{2 n+1}(c)$. In this article we consider the Legendrian submanifold $M$ is Ricci generalized pseudo-parallel in case of $n \geq 2$.
\end{abstract}

Mathematics Subject Classification: 53C15; 53C40; 53C25

Keywords: Legendrian submanifold; H-umbilical submanifold; Ricci generalized pseudo-parallel submanifold; Sasakian space form

\section{Introduction}

There are two typical submanifolds in an almost contact manifold: one is the class of C-totally real submanifolds and the other is the class of invariant submanifolds, which correspond to the class of totally real submanifolds and the class of holomorphic submanifolds in an almost Hermitian manifold,

\footnotetext{
${ }^{1}$ The author is supported by the NNSF 11071257 and partially by Science Foundation of China University of Petroleum (Beijing).
} 
respectively. A Sasakian manifold $\widetilde{M}$ is an almost contact manifold with normal almost contact structure $(\phi, \xi, \eta)$. If a Sasakian manifold $(\widetilde{M}, \phi, \xi, \eta)$ has constant $\phi$-sectional curvature then it is called a Sasakian space form. Concerning C-totally real submanifolds and invariant submanifolds of Sasakian space forms, there have been many results(see [9],[17],[11],[8]).

Recall that an isometric immersion $f: M^{n} \rightarrow \widetilde{M}^{n+k}$ from an $n$-dimensional Riemannian manifold into $n+k$-dimensional Riemannian with metric $g$ is pseudo-parallel if its second fundamental form $h$ satisfies

$$
\bar{R}(X, Y) \cdot h=L_{h} Q(g, h),
$$

where $\bar{R}(X, Y)$ is the curvature operator with respect to the van der WaerdenBortolotti connection $\bar{\nabla}$ of $f, L_{h}$ is some suitable smooth function on $M$. In particular, if $L_{h} \equiv 0, M$ is called a semi-parallel submanifold. The definition of pseudo-parallel was introduced in [1],[2] as a natural extension of semi-parallel and as the extrinsic analogue of pseudo-symmetry in the sense of Descz [7].

Recently, with respect to the study of pseudo-parallel immersion, many conclusions were showed (see $[1,2,12,13,14]$ ), where the ambient manifold $\widetilde{M}$ has constant sectional curvature. Otherwise, in [10], the authors studied the hypersurfaces in 4-dimensional space form $N^{4}(c)$ satisfying the curvature condition

$$
\bar{R}(X, Y) \cdot h=L Q(S, h) .
$$

Here $L$ is some smooth function on $M$ and $S$ denotes by the Ricci tensor of $M$. Such a hypersurface is called a Ricci generalized pseudo-parallel(see [1] and [2]). Specially, Yildiz et al. [18] considered Ricci generalized pseudoparallel Kähler submanifolds of complex space forms. They proved that if $M^{n}$ is Ricci generalized pseudo-parallel, then either $M$ is totally geodesic, or $\|h\|^{2}=-\frac{2}{3}\left(L \tau-\frac{1}{2}(n+2) c\right)$, or at some point $x$ of $M,\|h\|^{2}(x)>-\frac{2}{3}(L(x) \tau(x)-$ $\left.\frac{1}{2}(n+2) c\right)$.

On the other hand, we notice that the authors studied pseudo-parallel Lagrangian submanifolds of a complex space form in [5] and that A.Yildiz etc's results [19], where they considered the pseudo-parallel C-totally real minimal submanifolds in a Sasakian space form. Analogous to the Lagrangian submanifold in a complex space form, we call $M^{n}$ a Legendrian submanifold of a Sasakian space form $\widehat{M}^{2 m+1}(c)$ if $M$ is a C-totally real submanifold with $n=m$. In the present paper we consider Ricci generalized pseudo-parallel Legendrian submanifolds with flat normal bundle in a Sasakian space form. We show the following main theorem.

Theorem 1.1 Let $M^{n}(n \geq 2)$ be a Ricci generalized pseudo-parallel Legendrian submanifold of a Sasakian space form $\widetilde{M}^{2 n+1}(c)(c \neq-3)$ with flat normal bundle. Then either $M^{n}$ is minimal or $L=\frac{1}{n-1}$. 


\section{Basic concepts}

Let $\widetilde{M}^{2 m+1}$ be a $2 m+1$-dimensional Riemannian manifold. $\widetilde{M}$ is said to be an almost contact manifold if it is equipped with an almost contact structure $(\phi, \xi, \eta)$, where $\phi$ is a $(1,1)$-tensor field, $\xi$ a unit vector field, $\eta$ an one-form dual to $\xi$ satisfying

$$
\phi^{2}=-I+\eta \otimes \xi, \eta \circ \phi=0, \phi \circ \xi=0 .
$$

It is well-known that there exists a Riemannian metric $g$ such that

$$
\begin{gathered}
g(\phi X, \phi Y)=g(X, Y)-\eta(X) \eta(Y), \\
g(\phi X, Y)=-g(X, \phi Y), g(X, \xi)=\eta(X),
\end{gathered}
$$

where $X, Y \in \mathfrak{X}(\widetilde{M})$. Moreover, if the almost contact structure $(\phi, \xi, \eta)$ is normal, i.e.

$$
\left(\widetilde{\nabla}_{X} \phi\right) Y=g(X, Y) \xi-\eta(Y) X, \quad \widetilde{\nabla}_{X} \xi=-\phi X
$$

for all vector $X, Y$ on $T \widetilde{M}$, where $\widetilde{\nabla}$ denotes the connection with respect to $g$, then $\widetilde{M}$ is said to be a Sasakian manifold. For more details and background, see [4] and [16].

A plane of $T_{p} \widetilde{M}$ at $p$ is called $\phi$-section if it is spanned by $X$ and $\phi X$, where $X$ is orthonormal to $\xi$. The curvature of $\phi$-section is called $\phi$-sectional curvature.

A $2 m+1$-Sasakian space form is defined as a $2 m+1$-Sasakian manifold with constant $\phi$-sectional curvature $c$ and is denoted by $\widetilde{M}^{2 m+1}(c)$. As the examples of Sasakian space form, $\mathbb{R}^{2 m+1}$ and $S^{2 m+1}$ are equipped with Sasakian space form structures(more details in [3] and [16]). The curvature of a Sasakian space form $\widetilde{M}^{2 m+1}(c)$ is given by [16]

$$
\begin{aligned}
\widetilde{R}(X, Y) Z & =\frac{c+3}{4}(g(Y, Z) X-g(X, Z) Y) \\
& +\frac{c-1}{4}\{\eta(X) \eta(Z) Y-\eta(Y) \eta(Z) X+g(X, Z) \eta(Y) \xi \\
& -g(Y, Z) \eta(X) \xi+g(\phi Y, Z) \phi X-g(\phi X, Z) \phi Y-2 g(\phi X, Y) \phi Z\}
\end{aligned}
$$

for any $X, Y, Z \in T \widetilde{M}$.

Next we assume that $\widetilde{M}(c)$ is a Sasakian space form with constant $\phi$ sectional curvature $c \neq-3$. Let $f: M^{n} \rightarrow \widetilde{M}^{2 m+1}(c)$ be an isometric immersion of an $n$-dimensional manifold $M$ into $\widetilde{M}^{2 m+1}(c)$. If the unit vector field $\xi$ 
is normal to $M$, then we say $M$ is a $C$-totally real submanifold. It is well-known that for such a submanifold $\phi$ maps any tangent vector to $M$ at any $p \in M$ into the normal vector space $T_{p}^{\perp} M$, i.e. $\phi T_{p} M \subset T_{p}^{\perp} M$. In particular, when $n=m$, a C-totally real submanifold is called a Legendrian submanifold. In the other words, a Legendrian submanifold of a Sasakian space form $\widetilde{M}^{2 n+1}(c)$ is an antiinvariant submanifold with dimension $n$. Therefore we obtain from (2.4) and (2.5) that for any $X, Y \in T M, g(\phi X, \phi Y)=g(X, Y), \quad \eta(X)=g(X, \xi)=0$.

We define the endomorphism $X \wedge_{B} Y$ of $\mathfrak{X}(M)$ and the tensor $Q(B, T)$ for a $(0, k)$-tensor field $T, k \geq 1$ and a $(0,2)$-tensor field $B$ on $(M, g)$ by

$$
\begin{aligned}
\left(X \wedge_{B} Y\right) Z & =B(Y, Z) X-B(X, Z) Y, \\
Q(B, T)\left(X_{1}, \cdots, X_{k} ; X, Y\right) & =-T\left(\left(X \wedge_{B} Y\right) X_{1}, X_{2}, \cdots, X_{k}\right) \\
& -\cdots-T\left(X_{1}, \cdots, X_{k-1},\left(X \wedge_{B} Y\right) X_{k}\right),
\end{aligned}
$$

respectively. As usual, $\nabla$ and $\nabla^{\perp}$ denote by the Levi-Civita connection and the normal connection of $M$, respectively. Then the second fundamental form $h$ is given by the formula $h(X, Y)=\widetilde{\nabla}_{X} Y-\nabla_{X} Y$. Similarly, the Weingarten formula is: $\widetilde{\nabla}_{X} N=-A_{N} X+\nabla_{X}^{\perp} N$, where $N$ is an normal vector on $M$ and $A_{N}$ is the shape operator. The shape operator is related to the second fundamental form by

$$
g\left(A_{N} X, Y\right)=g(h(X, Y), N)=g\left(X, A_{N} Y\right) .
$$

The basic Gauss equation and Ricci equation are:

$$
\begin{aligned}
g(\widetilde{R}(X, Y) Z, W) & =g(R(X, Y) Z, W)+g(h(X, Z), h(Y, W))-g(h(X, W), h(Y, Z)), \\
g(\widetilde{R}(X, Y) N, V) & =g\left(R^{\perp}(X, Y) N, V\right)-g\left(\left[A_{N}, A_{V}\right] X, Y\right), \forall N, V \in T^{\perp} M,
\end{aligned}
$$

where $R$ and $R^{\perp}$ are curvature tensors corresponding to $\nabla$ and $\nabla^{\perp}$, respectively. The Codazzi equation: $(\widetilde{R}(X, Y) Z)^{\perp}=\left(\bar{\nabla}_{X} h\right)(Y, Z)-\left(\bar{\nabla}_{Y} h\right)(X, Z)$. Here $\bar{\nabla}=\nabla \oplus \nabla^{\perp}$ stands for the Van der Waerden-Bortoloti connection, given by

$$
\left(\bar{\nabla}_{X} h\right)(Y, Z)=\nabla_{X}^{\perp} h(Y, Z)-h\left(\nabla_{X} Y, Z\right)-h\left(Y, \nabla_{X} Z\right) .
$$

Note that the following facts are well-known:

Lemma 2.1 (see [9]) For a C-totally real submanifold, the following equations hold:

$$
\begin{aligned}
A_{\phi X} Y & =A_{\phi Y} X \\
A_{\phi X} Y & =-\phi h(X, Y)=A_{\phi Y} X, \quad A_{\xi}=0 \\
g(h(X, Y), \phi Z) & =g(h(X, Z), \phi Y) .
\end{aligned}
$$


Since a Legendrian submanifold is a special C-totally real submanifold, the above equations hold for a Legendrian submanifold. Therefore, it reduces from (2.3) and (2.12) that

$$
\phi A_{\phi X} Y=h(X, Y)=\phi A_{\phi Y} X
$$

Moreover, using (2.14) and (2.5), from the Gauss equation we get

$$
\widetilde{R}(X, Y)=R(X, Y)-\left[A_{\phi X}, A_{\phi Y}\right] .
$$

For an orthonormal frame $\left\{e_{1}, \cdots, e_{n}\right\}$ of $M$, the Ricci tensor $S$ of $M^{n}$ is defined by

$$
S(X, Y)=\sum_{i=1}^{n} g\left(R\left(e_{i}, X\right) Y, e_{i}\right)
$$

Assume that the normal bundle is flat, then we obtain from (2.6) and (2.15) that

$$
S(X, Y)=\frac{c+3}{4}\left\{n g(X, Y)-\sum_{i=1}^{n} g\left(e_{i}, X\right) g\left(e_{i}, Y\right)\right\}=\frac{c+3}{4}(n-1) g(X, Y) .
$$

For any vector fields $Z, W$ on $M$, the curvature operator $\bar{R}(X, Y)$ with respect to $\bar{\nabla}$ and $X \wedge_{S} Y$ can be extended as derivations of tensor fields. Thus, from (2.7) and (2.10) we have

$$
\begin{gathered}
(\bar{R}(X, Y) \cdot h)(Z, W)=\left(\bar{\nabla}_{X} \bar{\nabla}_{Y} h-\bar{\nabla}_{Y} \bar{\nabla}_{X} h-\bar{\nabla}_{[X, Y]} h\right)(Z, W) \\
=R^{\perp}(X, Y)(h(Z, W))-h(R(X, Y) Z, W)-h(Z, R(X, Y) W), \\
\left(X \wedge_{S} Y \cdot h\right)(Z, W)=-h\left(\left(X \wedge_{S} Y\right) Z, W\right)-h\left(Z,\left(X \wedge_{S} Y\right) W\right) \\
=-S(Y, Z) h(X, W)+S(X, Z) h(Y, W) \\
\\
\quad-S(Y, W) h(X, Z)+S(X, W) h(Y, Z) .
\end{gathered}
$$

By (2.14),(2.16) and (2.17), if the normal bundle is flat, i.e. $R^{\perp}=0$, then (1.2) becomes

$$
\begin{aligned}
-A_{\phi W} R(X, Y) Z & -A_{\phi Z} R(X, Y) W \\
= & L\left\{-S(Y, Z) A_{\phi X} W+S(X, Z) A_{\phi Y} W\right. \\
& \left.-S(Y, W) A_{\phi X} Z+S(X, W) A_{\phi Y} Z\right\} .
\end{aligned}
$$

That is, a Legendrian submanifold $M^{n}$ with flat normal bundle of $\widetilde{M}^{2 n+1}(c)$ is Ricci generalized pseudo-parallel if only if the equation (2.18) is satisfied. In particular, if $L \equiv 0$, then $M$ is called semi-parallel. It is obvious that a totally geodesic submanifold is semi-parallel. 


\section{The proof of Theorem 1.1}

For a constant curvature manifold if its normal bundle is flat, Cartan,E. proved the following well-known fact (see [6]):

Lemma 3.1 (Cartan,E) Let $M^{n}$ be a submanifold of constant curvature space $\widetilde{M}^{n+k}(c),\left\{\xi_{\alpha}\right\}$ local orthogonal normal vector fields, and $\left\{h^{\alpha}\right\}$ the second fundamental forms corresponding to $\left\{\xi_{\alpha}\right\}$. Then in every point of $M$, all $H^{\alpha}$ are mutually diagonalizable if and only if the normal bundle of $M$ is flat.

We know that for any vector $X, Y, Z \in T M$,

$$
R(X, Y) Z=\widetilde{R}(X, Y) Z=\frac{c+3}{4}(g(Y, Z) X-g(X, Z) Y .
$$

Therefore, by making use of (2.18) and (3.19), we have

$$
(1-(n-1) L)\left\{g(X, W) A_{\phi Y} Z-g(Y, Z) A_{\phi X} W+g(X, Z) A_{\phi Y} W-g(Y, W) A_{\phi X} Z\right\}=0 .
$$

By Lemma 3.1, for any $p \in M$ there exists a local orthogonal frame $\left\{e_{i}\right\}$ of $M^{n}$ such that all the second fundamental form tensors are mutually diagonalizable, namely, for any unit normal vector field $N, A_{N}\left(e_{i}\right)=\lambda_{i}^{N} e_{i}$, where $\lambda_{i}^{N}$ are the principle curvatures of $M$ with respect to $N$.

For a Legendrian submanifold $M$ we may choose an orthonormal basis of $T_{p}^{\perp} M$ of the form $\left\{e_{n+1}=\phi e_{1}, \cdots, e_{2 n}=\phi e_{n}, e_{2 n+1}=\xi\right\}$. For any $i, j \in$ $\{1, \cdots, n\}$, denote $\lambda_{i}^{n+j}$ by the principle curvature with respect to normal vector field $\phi e_{j}$, i.e.,

$$
A_{\phi e_{j}}\left(e_{i}\right)=\lambda_{i}^{n+j} e_{i}
$$

Thus in this case the mean curvature vector can be written as $H^{n+j}=\frac{1}{n} \sum_{i} \lambda_{i}^{n+j}$. In view of (3.20), setting $X=e_{i}, Y=e_{j}, Z=e_{k}$ and $W=e_{l}$, we have

$$
(1-(n-1) L)\left\{\delta_{i l} A_{\phi e_{j}} e_{k}-\delta_{j k} A_{\phi e_{i}} e_{l}+\delta_{i k} A_{\phi e_{j}} e_{l}-\delta_{j l} A_{\phi e_{i}} e_{k}\right\}=0,
$$

where $\delta_{i j}=g\left(e_{i}, e_{j}\right), 1 \leq i, j, k, l \leq n$. Using (3.21), we obtain

$$
(1-(n-1) L)\left\{\lambda_{k}^{n+j} \delta_{i l} e_{k}-\lambda_{l}^{n+i} \delta_{j k} e_{l}+\lambda_{l}^{n+j} \delta_{i k} e_{l}-\lambda_{k}^{n+i} \delta_{j l} e_{k}\right\}=0 .
$$

Moreover, we have

$$
(1-(n-1) L)\left\{\lambda_{k}^{n+j} \delta_{i l} \delta_{k s}-\lambda_{l}^{n+i} \delta_{j k} \delta_{l s}+\lambda_{l}^{n+j} \delta_{i k} \delta_{l s}-\lambda_{k}^{n+i} \delta_{j l} \delta_{k s}\right\}=0 .
$$

If we assume $j=s, i=k$ in (3.22) and sum over $j=1, \cdots, n$, it reduces to

$$
(1-(n-1) L)\left\{\lambda_{i}^{n+i} \delta_{i l}-\lambda_{l}^{n+i} \delta_{i l}+\lambda_{l}^{n+l}-\lambda_{i}^{n+i} \delta_{i l}\right\}=0
$$


Furthermore, by summing over $i=1, \cdots, n$, we have

$$
(1-(n-1) L)\left\{\sum_{i} \lambda_{i}^{n+i} \delta_{i l}-\lambda_{l}^{n+l}+n \lambda_{l}^{n+l}-\sum_{i} \lambda_{i}^{n+i} \delta_{i l}\right\}=0 .
$$

Because it follows from (2.11) that $\lambda_{i}^{n+l}=g\left(A_{\phi e_{l}} e_{i}, e_{i}\right)=g\left(A_{\phi e_{i}} e_{i}, e_{l}\right)=$ $\lambda_{i}^{n+i} \delta_{i l}$, so Eq.(3.23) implies

$$
[1-(n-1) L] \lambda_{l}^{n+l}=0 .
$$

On the other hand, using (3.21), we also get from (2.18) that $-\lambda_{s}^{n+l} R_{i j k s}-\lambda_{s}^{n+k} R_{i j l s}=L\left\{-\delta_{j k} \delta_{l s} \lambda_{l}^{n+i}+\delta_{i k} \delta_{l s} \lambda_{l}^{n+j}-\delta_{j l} \delta_{k s} \lambda_{k}^{n+i}+\delta_{i l} \delta_{k s} \lambda_{k}^{n+j}\right\}$.

Since

$$
R_{i j k s}=\frac{c+3}{4}\left(\delta_{j k} \delta_{i s}-\delta_{i k} \delta_{j s}\right), \quad R_{i j l s}=\frac{c+3}{4}\left(\delta_{j l} \delta_{i s}-\delta_{i l} \delta_{j s}\right),
$$

by substituting (3.26) into (3.25), we get

$$
\begin{aligned}
& -\left\{\lambda_{s}^{n+l}\left(\delta_{j k} \delta_{i s}-\delta_{i k} \delta_{j s}\right)+\lambda_{s}^{n+k}\left(\delta_{j l} \delta_{i s}-\delta_{i l} \delta_{j s}\right)\right\} \\
& \quad=(n-1) L\left\{-\delta_{j k} \delta_{l s} \lambda_{l}^{n+i}+\delta_{i k} \delta_{l s} \lambda_{l}^{n+j}-\delta_{j l} \delta_{k s} \lambda_{k}^{n+i}+\delta_{i l} \delta_{k s} \lambda_{k}^{n+j}\right\} .
\end{aligned}
$$

In the same way, putting $i=k, j=s$ and summing over $i=1, \cdots, n, j=$ $1, \cdots, n$ in $(3.27)$, respectively, we have

$$
H^{n+l}=(n-1) L \lambda_{l}^{n+l} .
$$

Combing (3.28) with (3.24), we concluded that if $L=0$ then $H^{n+l}=0$ for any $1 \leq l \leq n$, that is, $M$ is minimal. if $L \neq 0$, then $\frac{1-(n-1) L}{(n-1) L} H^{n+l}=0$, which implies $H^{n+l}=0$ or $L=\frac{1}{n-1}$. It completes the proof of Theorem.

Recall that an non-totally geodesic Legendrian $\mathrm{H}$-umbilical submanifold $M^{n}$ of Sasakian manifold $\widetilde{M}^{2 n+1}$ is a Legendrian submanifold and its second fundamental form satisfies the following forms:

$$
\begin{gathered}
h\left(e_{1}, e_{1}\right)=\lambda \phi e_{1}, h\left(e_{2}, e_{2}\right)=\cdots=h\left(e_{n}, e_{n}\right)=\mu \phi e_{1}, \\
h\left(e_{1}, e_{j}\right)=\mu \phi e_{j}, h\left(e_{j}, e_{k}\right)=0,2 \leq j \neq k \leq n,
\end{gathered}
$$

for some suitable functions $\lambda$ and $\mu$ with respect to some suitable orthonormal local frame field $\left\{e_{i}\right\}$ of $M[15]$. We have the following corollary:

Corollary 3.2 For $c \neq-3$, assume that $M^{n}$ is a Legendrian H-umbilical submanifold of a Sasakian space form $\widetilde{M}^{2 n+1}(c)$ with flat normal bundle. If $M^{n}$ is Ricci generalized pseudo-parallel then either $L=\frac{1}{n-1}$, or $n=1$. 
Proof. We consider $\left\{e_{1}, \cdots, e_{n}\right\}$ as (3.29), then from (3.20) we get

$$
(1-(n-1) L)\left\{\delta_{i l} A_{\phi e_{j}} e_{k}-\delta_{j k} A_{\phi e_{i}} e_{l}+\delta_{i k} A_{\phi e_{j}} e_{l}-\delta_{j l} A_{\phi e_{i}} e_{k}\right\}=0 .
$$

Assume that $j=1$ and $i=k$ in (3.30), a straightforward calculation implies

$$
(1-(n-1) L)\left\{(n-1)(\lambda-\mu) e_{1}+n \mu \sum_{l=2}^{n} e_{l}\right\}=0
$$

If $L \neq \frac{1}{n-1}$, then $(n-1)(\lambda-\mu) e_{1}+n \mu \sum_{l=2}^{n} e_{l}=0$. Since $\left\{e_{i}\right\}$ is orthonormal, it means that $\mu=0$ and $(n-1)(\lambda-\mu)=0$. Therefore $n=1$. $\square$

\section{References}

[1] A.C. Asperti, G.A. Lobos and F. Mercuri, Pseudo-parallel immersions in space forms, Math. Contemp. 17(1999), 59-70.

[2] A.C. Asperti, G.A. Lobos and F. Mercuri, Pseudo-parallel submanifolds of a space form, Adv. Geom. 2(2002), 57-71.

[3] D.E. Blair, Rimemannian Geometry of Contact and Symplectic Manifolds. Birkhäuser, 2002.

[4] D.E. Blair, Contact manifolds in Riemannian Geometry. Lect Notes Math 509. Berlin Heibelberg New York: Springer, 1976.

[5] P. M. Chacón and G.A.Lobos, Pseudo-parallel Lagrangian submanifolds in complex space forms, Differential Geometry and its Applications $27(2009)$, 137-145.

[6] B. Y. Chen, Geometry of Submanifolds. New York: Dekker, 1973.

[7] R. Deszcz, On pseudosymmetric spaces, Bull. Belg. Math. Soc. 44(1992), $1-34$.

[8] F. Dillen, I. Mihai and L. Varancken, A class of C-totally real submanifolds of Sasakian space forms, J. Austral. Math. Soc.(Series A) 64(1998), 120-128.

[9] F. Dillen and L. Varancken, C-totally real submanifolds of Sasakian space forms, J. Math. Pure. Appl. 69(1990), 85-93.

[10] R. Deszcz, L. Verstraelen and S. Yaprak, Pseudosymmetric Hypersurfaces in 4-dimensional Space of Constant Curvature, Bull. Ins. Math. Acad. Sinica 22(1994),167-179. 
[11] X. M. Liu, On Ricci curvature of C-totally real submanifolds in Sasakian space forms, Pro. Indian Acad. Sci.(Math. Sci.) 111,no.4(2001), 399-405.

[12] G.A. Lobos, Pseudo-parallel surfaces in space forms, in: Differential Geometry, Valencia, 2001, World Sci. Publishing, River Edger, NJ, 2002, pp. 197-204.

[13] G.A. Lobos and M. Ortega, Pseudo-parallel real hypersurfaces in complex space forms. Bull. Korean. Math. Soc. 41(2004), 609-618.

[14] G.A. Lobos and R. Tojeiro, Pseudo-parallel submanifolds with flat normal bundle of space forms, Glasg. Math. J. 48(2006), 171-177.

[15] I. Mihai and I.N. Rădulescu, An improved Chen-Ricci inequality for Legendrian submanifolds in Sasakian space forms. J. Adv. Math. Stud. 4,no 2(2011), 51-58.

[16] K. Yano and M. Kon, Structures on Manifolds. world Scientific: Singapore, 1984.

[17] A. Yildiz and C. Muranthan, Invariant submanifolds of Sasakian space forms, J. Geom. 95(2009),135-150.

[18] A. Yildiz and C. Muranthan, Ricci generalized pseudo-parallel Kähler submanifolds in complex space forms, Bull. Malays. Sci. Soc. (2)31(2)(2008), 153-163.

[19] A. Yildiz, C. Muranthan, K. Arslan and R. Ezentas, C-totally real pseudo-parallel submanifolds of Sasakian space forms, Monatsh. Math. 151(2007), 247-256.

\section{Received: April 3, 2014}

